\title{
Graphene as a Strictly 2D Sheet or as a Film of Small but Finite Thickness
}

\author{
Bo E. Sernelius \\ Division of Theory and Modeling, Department of Physics, Chemistry, and Biology, Linköping University, \\ Linköping, Sweden \\ Email: bos@ifm.liu.se
}

Received July 13, 2012; revised August 13, 2012; accepted September 30, 2012

\begin{abstract}
We study an interface between two media separated by a strictly 2D sheet. We show how the amplitude reflection coefficient can be modeled by that for an interface where the 2D sheet has been replaced by a film of small but finite thickness. We give the relationship between the 3D dielectric function of the thin film and the 2D dielectric function of the sheet. We apply this to graphene and show how the van der Waals interaction between two graphene sheets is modified when going from the 2D sheet description to the thin film description. We also show the wrong result from keeping the 2D dielectric function to represent the film medium.
\end{abstract}

Keywords: Graphene; 2D Sheet; Thin Film; Casimir; Van Der Waals

\section{Introduction}

There are no strictly 2D (two-dimensional) systems in the real world. However, if the carriers are strongly confined in one direction they have quantized energy levels for one spatial dimension but are free to move in two spacial dimensions [1]. Thus the wave vector is a good quantum number for two dimensions but not for the third. Polarizability for 2D systems was discussed for the first time by F. Stern [2]. Graphene is a good example of a 2D system. The dielectric function of grapheme [3-7] has been derived in the literature. This function is valid for an idealized 2D sheet. From a distance this idealization is a valid approximation but at close proximity of the sheet the finite thickness of the real atomic layer may influence the physics. One feasible approximation is to represent the layer by a homogeneous film of small but finite thickness. The dielectric function of the film is not represented by the 2D dielectric function. This has to be modified into a 3D version. How this modification is done is what this work is all about. The general results we obtain are not limited to graphene. We use graphene as an illustration. Examples where the results are useful are for optical properties of layered structures and for dispersion interactions (van der Waals and Casimir) in layered structures. The amplitude reflection coefficient for p-polarization was given for the first time in graphene mono- and multi-layers by Falkovsty et al. [8] for the far-infrared, and by Stauber et al. [9] for the visible region of the spectrum.
In Section 2 we give the amplitude reflection coefficients for an interface between two media, in Section 2.1 we show how these are modified when a $2 \mathrm{D}$ sheet is inserted at the interface, in Section 2.2 we show how these are modified when instead a film of finite thickness is inserted, and in Section 2.3 we show how a 2D sheet can be modeled by a homogeneous film of finite thickness. In Section 3 we illustrate this modeling for graphene. Before we end with a brief summary and conclusion Section, 5, we discuss in Section 4 how the results for 2D sheets and thin films can be used in non-planar structures.

\section{Amplitude Reflection Coefficient at an Interface}

For the present task we need a geometry consisting of two regions and one interface, $i \mid j$. For planar structures there are two types of mode [10], transverse magnetic (TM) or $p$-polarized and transverse electric (TE) or $s$-polarized. They have different amplitude reflection coefficients. At an interface between medium $i$ and $j$ the TM and TE amplitude reflection coefficients for waves impinging from the $i$ side are

$$
r_{i j}^{T M}=\frac{\tilde{\varepsilon}_{j} \gamma_{i}-\tilde{\varepsilon}_{i} \gamma_{j}}{\tilde{\varepsilon}_{j} \gamma_{i}+\tilde{\varepsilon}_{i} \gamma_{j}}
$$

and

$$
r_{i j}^{T E}=\frac{\gamma_{i}-\gamma_{j}}{\gamma_{i}+\gamma_{j}}
$$


respectively. Note that $r_{j i}=-r_{i j}$ holds for both mode types. If retardation is neglected there is only one mode type and the amplitude reflection coefficient is

$$
r_{i j}=\frac{\tilde{\varepsilon}_{j}-\tilde{\varepsilon}_{i}}{\tilde{\varepsilon}_{j}+\tilde{\varepsilon}_{i}}
$$

In the above equations $\gamma_{i}=\sqrt{1-\tilde{\varepsilon}_{i}(\omega)(\omega / c k)^{2}}$, $\tilde{\varepsilon}_{i}(\omega)$ is the dielectric function of medium $i, c$ is the speed of light in vacuum, and $k$ is the length of a wave vector in the plane of the interface. We have suppressed the arguments of the functions in Equations (1)-(3). The amplitude reflection coefficients and the $\gamma$-functions are functions of $k$ and $\omega$. The dielectric functions are functions of $\omega$, only, i.e. spatial dispersion is neglected. Inclusion of spatial dispersion in the bulk dielectric functions is possible $[11,12]$ but would lead to much higher complexity and negligible effects for the present problem.

\subsection{Interface with a Strictly 2D Sheet}

There are different formulations of electromagnetism in the literature. The difference lies in how the conduction carriers are treated. In one formulation these carriers are lumped together with the external charges to form the group of free charges. Then only the bound charges contribute to the screening. We want to be able to treat geometries with metallic regions. Then this formulation is not suitable. In the formulation that we use the conduction carriers are treated on the same footing as the bound charges. Thus, both bound and conduction charges contribute to the dielectric function. In the two formalisms the $\boldsymbol{E}$ and $\boldsymbol{B}$ fields, the true fields, of course are the same. However, the auxiliary fields the $\boldsymbol{D}$ and $\boldsymbol{H}$ fields are different. To indicate that we use this alternative formulation we put a tilde above the $\boldsymbol{D}$ and $\boldsymbol{H}$ fields and also above the dielectric functions. See [6] for a fuller discussion on this topic. Within this formalism the standard boundary conditions, used to derive the reflection coefficients, are that in absence of external charge and current densities at an interface the tangential components of the $\boldsymbol{E}$ and $\tilde{\boldsymbol{H}}$ fields and the normal components of the $\tilde{\boldsymbol{D}}$ and $\boldsymbol{B}$ fields are all continuous across the interface. The sources to the fields in Maxwell's equations are the external charge and current densities. In the boundary conditions any discontinuities in the normal component of the $\tilde{\boldsymbol{D}}$ fields and tangential component of the $\tilde{\boldsymbol{H}}$ fields are caused by external surface charge densities and external surface current densities, respectively.

The amplitude reflection coefficient gets modified if there is a $2 \mathrm{D}$ layer at the interface. We treat [6] the $2 \mathrm{D}$ layer at the interface as external to our system. The modified amplitude reflection coefficient for a TM mode is [6]

$$
r_{i j}^{T M}=\frac{\tilde{\varepsilon}_{j} \gamma_{i}-\tilde{\varepsilon}_{i} \gamma_{j}+2 \gamma_{i} \gamma_{j} \alpha^{\|}}{\tilde{\varepsilon}_{j} \gamma_{i}+\tilde{\varepsilon}_{i} \gamma_{j}+2 \gamma_{i} \gamma_{j} \alpha^{\|}}
$$

where the polarizability, $\alpha^{\square}$, of the 2D sheet is obtained from the dynamical conductivity, $\sigma$,

$$
\alpha^{\square}(k, \omega)=\frac{2 \pi i \sigma(k, \omega) k}{\omega}
$$

and the dielectric function is

$$
\varepsilon^{\square}(k, \omega)=1+\alpha(k, \omega)
$$

For TM modes the tangential component of the electric field, which will induce the external current, is parallel to $\boldsymbol{k}$, so the longitudinal 2D dielectric function of the sheet enters. The bound charges in the $2 \mathrm{D}$ sheet also contribute to the dynamical conductivity and the polarizability.

The modified amplitude reflection coefficient for a TE mode is [6]

$$
r_{i j}^{T E}=\frac{\gamma_{i}-\gamma_{j}+2(\omega / c k)^{2} \alpha^{\perp}}{\gamma_{i}+\gamma_{j}-2(\omega / c k)^{2} \alpha^{\perp}}
$$

where the polarizability, $\alpha^{\perp}$, of the 2D sheet is obtained from the dynamical conductivity, $\sigma^{\perp}$,

$$
\alpha^{\perp}(k, \omega)=\frac{2 \pi i \sigma^{\perp}(k, \omega) k}{\omega}
$$

and the dielectric function is

$$
\varepsilon^{\perp}(k, \omega)=1+\alpha^{\perp}(k, \omega)
$$

For a TE wave the electric field is perpendicular to $\boldsymbol{k}$, so the transverse $2 \mathrm{D}$ dielectric function of the sheet enters.

If retardation is neglected there is only one mode type and the amplitude reflection coefficient is

$$
r_{i j}=\frac{\tilde{\varepsilon}_{j}-\tilde{\varepsilon}_{i}+2 \alpha^{\|}}{\tilde{\varepsilon}_{j}+\tilde{\varepsilon}_{i}+2 \alpha^{\|}}
$$

Now we have in Equations (4), (7), and (10) the amplitude reflection coefficients for an interface between two media with a 2D sheet sandwiched in between. To be noted is that spatial dispersion of the $2 \mathrm{D}$ sheet can be included without any complications. This spatial dispersion can have important effects [13-16]. In next section we will show the corresponding results when instead of a $2 \mathrm{D}$ sheet we have a thin film sandwiched between the two media.

\subsection{Interface with a Film of Finite Thickness}

For the present task we need a geometry consisting of three regions and two interfaces, $i|j| k$.

For this composite interface the amplitude reflection 
coefficient for a wave impinging from the $i$ side is $[10,17]$

$$
r_{i j k}=\frac{r_{i j}+e^{-2 \gamma_{j} k d_{j}} r_{j k}}{1+e^{-2 \gamma_{j} k d_{j}} r_{i j} r_{j k}}
$$

where $d_{\mathrm{j}}$ is the thickness of the film $j$. This expression is valid for TM- and TE-modes when retardation is included and also for the modes when retardation is neglected. The appropriate amplitude reflection coefficient from Equations (1)-(3) should be used in the expression on the right hand side. In next section we show how the effect of a strictly 2D sheet can be modeled by a film of finite thickness with the proper choice of $3 \mathrm{D}$ dielectric function.

\subsection{Simulating a Strictly $2 D$ Film with a film of Finite Thickness}

Let us study a 2D-sheet placed in a time varying electric field, $\boldsymbol{E}$, in the plane of the film. There will be a surface current density, $\boldsymbol{K}=\sigma^{2 D} \boldsymbol{E}$. Since we want to treat this $2 \mathrm{D}$ sheet as a thin film of finite thickness, $\delta$, we let this current be spread evenly through the thickness of the film. The volume current density, $\boldsymbol{j}$, is then $\boldsymbol{j}=\boldsymbol{K} / \delta$ and since $\boldsymbol{j}=\sigma^{3 D} \boldsymbol{E}$ it follows that

$$
\sigma^{3 D}=\sigma^{2 D} / \delta
$$

Now, since

$$
\alpha^{2 D}=2 \pi i \sigma^{2 D} k / \omega
$$

and

$$
\alpha^{3 D}=4 \pi i \sigma^{3 D} / \omega
$$

we find that

$$
\alpha^{3 D}=2 \alpha^{2 D} / k \delta
$$

Thus, in problems with $2 \mathrm{D}$ sheets one can treat the sheets as thin 3D films where the 3D polarizability above is used. To check if this is a reasonable approach we insert the expressions in Equations (1)-(3) into Equation (11) where now $d_{\mathrm{j}}$ is $\delta$ and $\tilde{\varepsilon}_{j}=1+2 \alpha^{2 D} / k \delta$. If we now let the film thickness, $\delta$, go towards zero we reproduce the results in Equations (4), (7), and (10). Thus in the limit the model is exact.

\section{Graphene as an Illustrative Example}

We will now calculate the Casimir interaction energy between two free standing undoped graphene sheets in vacuum. To make it as simple as possible we neglect retardation and perform the calculations for zero temperature. Retardation effects are actually very small in graphene $[6,18]$.

In a general point, $z$, in the complex frequency plane, away from the real axis the polarizability is [3]

$$
\alpha^{2 D}(k, z)=\frac{2 \pi e^{2} g}{16 \hbar} \frac{k}{\sqrt{v^{2} k^{2}-z^{2}}}
$$

where $v$ is the carrier velocity which is a constant in graphene ( $E= \pm \hbar v k$ ), and $g$ represents the degeneracy parameter with the value of 4 (a factor of 2 for spin and a factor of 2 for the cone degeneracy). In the numerical calculations we use the value [4] $8.73723 \times 10^{5} \mathrm{~m} / \mathrm{s}$ for $v$. The polarizability in Equation (16) is valid for $\mathrm{T}=0$ when only the contributions from the Dirac cones are included and there is no gap at the Fermi level. Effects of finite temperature and modifications due to a gap, that in some situations open up, can be dealt with [19] but then one loses the advantage of having the expression on closed analytical form.

If we now treat the graphene sheet as a thin film of thickness $\delta$ the polarizability of the film material should be chosen as

$$
\alpha^{3 D}(k, z)=\frac{\pi e^{2}}{\hbar \delta} \frac{1}{\sqrt{v^{2} k^{2}-z^{2}}}
$$

and on the imaginary frequency axis it is

$$
\alpha^{3 D}(k, i \omega)=\frac{\pi e^{2}}{\hbar \delta} \frac{1}{\sqrt{v^{2} \mathrm{k}^{2}+\omega^{2}}}
$$

The van der Waals (vdW) and Casimir interactions can be derived in many different ways.

One way is to derive the interaction in terms of the electromagnetic normal modes [10] of the system. For planar structures the interaction energy per unit area can be written as [10]

$$
E=\hbar \int \frac{d^{2} k}{(2 \pi)^{2}} \int_{0}^{\infty} \frac{d \omega}{(2 \pi)} \ln \left[f_{k}(i \omega)\right]
$$

where $f_{k}\left(\omega_{k}\right)=0$ is the condition for electromagnetic normal modes. For the present geometry the mode condition function is

$$
f_{k}=1-e^{-2 k d}\left(r_{121}\right)^{2}
$$

where the index 1 stands for vacuum and the index 2 for the film material.

Using Equation (11) with the proper functions for our problem inserted we get

$$
r_{121}=\frac{r_{12}+e^{-2 k \delta} r_{21}}{1+e^{-2 k \delta} r_{12} r_{21}}=\frac{r_{12}\left(1-e^{-2 k \delta}\right)}{1-e^{-2 k \delta} r_{12}^{2}}
$$

where

$$
r_{12}=\frac{\alpha^{3 D}(k, i \omega)}{\alpha^{3 D}(k, i \omega)+2}
$$

For strictly $2 \mathrm{D}$ sheets the corresponding mode condi- 
tion function is

$$
f_{k}=1-e^{-2 k d}\left[\frac{\alpha^{2 D}(k, i \omega)}{1+\alpha^{2 D}(k, i \omega)}\right]^{2}
$$

The result of Equation (19) with the mode condition function from Equation (23) is shown as the solid curve in Figure 1. With the particular screening in graphene it turns out that $\alpha^{2 D}(k / \lambda, i \omega / \lambda)=\alpha(k, i \omega)$ and the separation dependence of the nonretarded interaction becomes very simple. A change in dummy variables removes the only $d$ in the integrand and produces the factor of $d^{-3}$ in front of the integral. The result is a straight line in Figure 1 with the power law $d^{3}$. The dashed curve in Figure 1 is the result one obtains if the 2D version of the polarizability, $\alpha^{2 D}$, is used instead of the $3 \mathrm{D}$ version, $\alpha^{3 D}$, to represent the film medium. As we see this wrong result is quite different. Even a different power law is obtained for large separations.

When we treat the graphene sheets as thin films of thickness $\delta$ the momentum, $k$, enters in more places in the integrand with the effect that the result has a more complicated dependence on $d$. However in those additional places $k$ always enters in the combination $k \delta$. This means that there will be a universal correction factor, from treating the sheet as a film, that depends on $d / \delta$. This correction factor is shown as the solid curve in Figure 2. One would expect the interaction to decrease for small separations since all matter in the two films is further apart than $d$, as can be seen in the inset of Figure 2. This expected reduction is also found but then there is an unexpected over shoot at larger distances with a maximum of $19 \%$ at around $8 \delta$. The dashed curve is the erroneous correction factor obtained if $\alpha^{2 D}$ instead of $\alpha^{3 D}$ is used. Unfortunately this was used in [20].

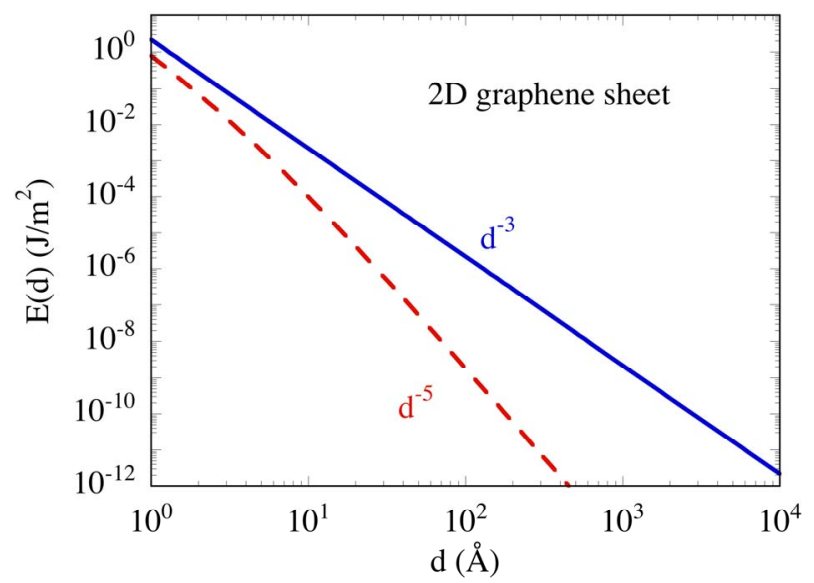

Figure 1. The attractive nonretarded [7] interaction energy per unit area between two graphene sheets as function of separation, solid curve. The dashed curve is the erroneous result obtained for a $1 \AA$ thick film when the unmodified graphene 2D polarizability is used for the film medium.

\section{Non-Planar Structures}

In this section we discuss how one may proceed in nonplanar structures, One may, e.g. have a cylinder or a sphere coated by a graphene or graphene-like film. The spatial dispersion complicates things. In these structures the momentum, $k$, is no longer a good quantum number for the normal modes. However, the problems are often dominated by long wavelengths. The $3 \mathrm{D}$ polarizability for a graphene film in the long wavelength limit is

$$
\alpha^{3 D}(i \omega) \approx \frac{\pi \mathrm{e}^{2}}{\hbar \delta \omega}
$$

Fortunately the wave number is now absent from the expression and nothing hinders the use of this expression in non-planar structures.

\section{Summary and Conclusions}

We have shown a way to modify the dielectric function when a homogeneous film of finite thickness is used to simulate a strictly 2D sheet or vice versa. As an illustration we calculated the van der Waals interaction between two undoped graphene sheets. The particular screening in graphene leads to a very simple van der Waals interacttion which follows a pure power law, the same power law as that for the Casimir interaction between two ideal metal half spaces. From this follows that the retardation effects are virtually absent. The general results presented here are valid for any 2D sheet, e.g. a 2D electron gas. In that case the spatial dispersion effects are more complicated [13-16].

We found a universal correction factor, as function of $\mathrm{d} / \delta$, to the interaction between the graphene sheets when they were modeled by films of thickness $\delta$. There was a maximum of $19 \%$ over shoot at separations around $8 \delta$.

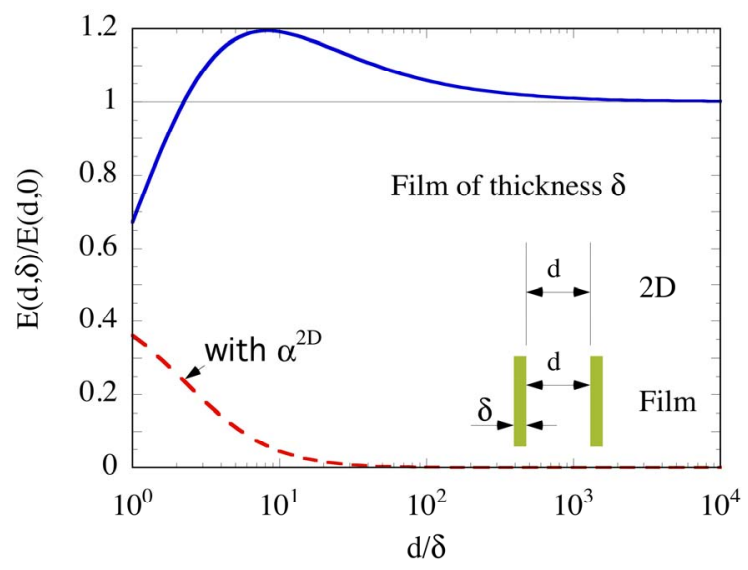

Figure 2. The ratio between the film result and the $2 \mathrm{D}$ sheet result for the interaction energy between two graphene sheets as function of the ratio between the separation and the film thickness, solid curve. The dashed curve is the erroneous result obtained when the unmodified $2 D$ polarizability is used for the film medium. 
We further pointed out how to proceed in the case of non-planar structures as to avoid problems caused by spatial dispersion.

\section{Acknowledgements}

We are grateful for financial support from the Swedish Research Council, VR Contract No. 70529001.

\section{REFERENCES}

[1] T. Ando, A. B. Fowler and F. Stern, "Electronic Properties of Two-Dimensional Systems," Reviews of Modern Physics, Vol. 54, No. 2, 1982, pp. 437-672. doi:10.1103/RevModPhys.54.437

[2] F. Stern, "Polarizability of a Two-Dimensional Electron Gas," Physical Review Letters, Vol. 18, No. 14, 1967, pp. 546-548. doi:10.1103/PhysRevLett.18.546

[3] J. Gonzáles, F. Guinea and M. A. H. Vozmediano, "Non-Fermi Liquid Behavior of Electrons in the HalfFilled Honeycomb Lattice (A Renormalization Group Approach)," Nuclear Physics B, Vol. 424, No. 3, 1994, pp. 595-618.

[4] B. Wunsch, T. Stauber, F. Sols and F. Guinea, "Dynamical Polarization of Graphene at Finite Doping," New Journal of Physics, Vol. 8, 2006, p. 318. doi: 10.1088/1367-2630/8/12/318

[5] E. H. Hwang, and S. Das Sarma, "Dielectric Function, Screening, and Plasmons in Two-Dimensional Graphene," Physical Review B, Vol. 75, No. 20, 2007, p. 205418. doi:10.1103/PhysRevB.75.205418

[6] B. E. Sernelius, "Retarded Interactions in Graphene Systems," Physical Review B, Vol. 85, No. 19, 2012, p. 195427. doi:10.1016/0550-3213(94)90410-3

[7] B. E. Semelius, "Casimir Interactions in Graphene Systems" Europhysics Letters, Vol. 5, No. 5, 2011, Article ID: 57003. doi:10.1209/0295-5075/95/57003

[8] L. A. Falkovsty and S. S. Pershoguba, "Optical Far-Infrared Properties of a Graphene Monolayer and Multilayer," Physical Review B, Vol. 76, No. 15, 2007, Article ID: 153410. doi:10.1103/PhysRevB.76.153410

[9] T. Stauber, N. M. R. Peres and A. K. Geim, "Optical Conductivity of Graphene in the Visible Region of the Spectrum," Physical Review B, Vol. 78, No. 8, 2008, Ar- ticle ID: 085432. doi:10.1103/PhysRevB.78.085432

[10] B. E. Sernelius, "Surface Modes in Physics," Wiley-VCH, Berlin, 2001.

[11] B. E. Sernelius, "Effects of Spatial Dispersion on Electromagnetic Surface Modes and on Modes Associated with a Gap between Two Half Spaces," Physical Review $B$, Vol. 71, No. 23, 2005, Article ID: 235114. doi:10.1103/PhysRevB.71.235114

[12] R. Esquivel and V. B. Svetovoy "Correction to the Casimir Force Due to the Anomalous Skin Effect," Physical Review A, Vol. 69, No. 6, 2004, Article ID: 062102. doi:10.1103/PhysRevA.69.062102

[13] B. E. Sernelius and P. Björk, "Interaction Energy for a Pair of Quantum Wells," Physical Review B, Vol. 57, No. 11, 1998, p. 6592. doi:10.1103/PhysRevB.57.6592

[14] M. Boström and Bo E. Sernelius, "Fractional van der Waals Interaction between Thin Metallic Films," Physical Review B, Vol. 61, No. 3, 2000, p. 2204. doi:10.1103/PhysRevB.61.2204

[15] J. F. Dobson, A. White and A. Rubio, "Asymptotics of the Dispersion Interaction: Analytic Benchmarks for van der Waals Energy Functionals," Physical Review Letters, Vol. 96, No. 7, 2006, Article ID: 073201. doi:10.1103/PhysRevLett.96.073201

[16] Bo E. Sernelius, "Casimir Effects in Graphene Systems: Unexpected Power Laws," International Journal of Modern Physics: Conference Series, Vol. 14, 2012, p. 531. doi:10.1142/S2010194512007660

[17] J. Mahanty and B. W. Ninham, "Dispersion Forces," Academic Press, London, 1976.

[18] G. Gómez-Santos, "Thermal van der Waals Interaction between Graphene Layers," Physical Review B, Vol. 80, 2009, Article ID: 245424. doi:10.1103/PhysRevB.80.245424

[19] V. Svetovoy, Z. Moktadir, M. Elwenspoek, and H. Mizuta, "Tailoring the Thermal Casimir Force with Graphene," Europhysics Letters, Vol. 96, No. 1, 2011, Article ID: 14006. doi:10.1209/0295-5075/96/14006

[20] Jalal Sarabadani, Ali Naji, Reza Asgari, and Rudolf Podgornik, "Many-Body Effects in the van der WaalsCasimir Interaction between Graphene Layers," Physical Review B, Vol. 84, No. 15, 2011, Article ID: 155407. doi:10.1103/PhysRevB.84.155407 